\title{
Photo-assisted Intersystem Crossing: The predominant triplet formation mechanism in some Isolated Polycyclic Aromatic Molecules excited with pulsed lasers
}

Philip M. Johnson ${ }^{a, \dagger}$ and Trevor J. Sears ${ }^{\dagger, \ddagger}$

'Department of Chemistry, Stony Brook University, Stony Brook, New York 11794-3400, United States

${ }^{\ddagger}$ Department of Chemistry, Brookhaven National Laboratory, Upton, New York 11973, United States

\section{ABSTRACT}

Naphthalene, anthracene, and phenanthrene are shown to have very long-lived triplet lifetimes when the isolated molecules are excited with nanosecond pulsed lasers resonant with the lowest singlet state. For naphthalene, triplet state populations are created only during the laser pulse, excluding the possibility of normal intersystem crossing at the one photon level, and all molecules have triplet lifetimes greater than hundreds of microseconds, similar to the behavior previously reported for phenylacetylene. Although containing 7 to 12 thousand $\mathrm{cm}^{-1}$ of vibrational energy, the triplet molecules have ionization thresholds appropriate to vibrationless $T_{1}$ states. The laser power dependences (slopes of log-log power plots) of the excited singlet and triplet populations are about 0.7 for naphthalene and about 0.5 for anthracene. Kinetic modeling of the power dependences successfully reproduces the experimental results and suggests that the triplet formation mechanism involves an enhanced spin orbit coupling caused by sigma character in states at the 2-photon level. Symmetry Adapted ClusterConfiguration Interaction calculations produced excited state absorption spectra to provide guidance for estimating kinetic rates and the sigma character present in higher electronic states. It is concluded that higher excited state populations are significant when larger molecules are excited with pulsed lasers and need to be taken into account whenever discussing the molecular photodynamics.

\footnotetext{
a Corresponding Author: E-mail; Philip.johnson@stonybrook.edu; telephone (631) 632-7912.
} 


\section{INTRODUCTION}

When energy is deposited into a molecule via a photon causing a transition to an excited electronic state, initially that new energy exists mostly as electronic energy, with only small amounts of rotational and vibrational being created by the photon. If a molecule is large enough, even if the photon is not energetic enough to cause photodissociation, the electronic energy can be partially or totally transformed into vibrational energy in a process called a radiationless transition. The quantum mechanical basis for describing the details of this energy transformation has been studied extensively ${ }^{1}$, and it has been found that a key element in determining the behavior of the particular excited states involved in the transition is the density of states of the receiving manifold at the energy of the initial state. Because the density of vibrational states at a given location in a vibrational well goes up extremely quickly with the number of atoms in the molecule, excited state behavior has been categorized as being in either the small molecule limit or the large molecule limit, along with a very interesting intermediate case, where what happens depends dramatically on the details of the excitation.

Of these three cases, it has generally been considered that the large molecule case was in many respects the simplest. The density of states in the vibrational manifolds at the energies being considered is so high that the states formed essentially a continuum, and the transition rates are averaged over so many possibilities that quantum mechanical considerations were able to be disregarded. Classical kinetic schemes have been fully capable of describing the observed phenomena.

When the theories of radiationless transitions were being developed, almost all of the experiments examining how molecules evolve in their excited states were done by recording the fluorescence emission from the samples. For larger molecules (10 or so atoms or more), it was found that if a radiationless transition was from the photon-coupled state into an electronic state lower by a substantial amount (the energy gap was large), the fluorescence decay was always exponential, and 
could be described as being in competition with other processes that converted energy into vibrations without photon emission.

In all discussions of radiationless transitions, one needs to keep in mind the environment of the molecules in question. In solids and liquids there are phonon modes which provide a vast bath that can irreversibly receive vibrational energy from the excited molecule, and external forces that can change the interstate couplings involved in the excited state evolution. In gases with higher temperatures and densities, collisions can play a role by inducing energy transfer as well as external perturbations in the couplings. In environments where the target molecules are completely isolated (very rarified gases, supersonic beams, and outer space), when a molecule is electronically excited it has no way to rid itself of energy except by radiation. Fluorescence is a major decay route, but in the laboratory, infrared emission and phosphorescence generally take too long and are not generally observed on the time scale of the experiment. Therefore, except for fluorescence, isolated molecule excited states evolve only by rearranging the energies contained in their various electronic and vibrational modes, without any loss of total energy. Usually the exciting photon deposits energy mostly into a single electronic mode, but the vastly greater number of vibrational degrees of freedom in larger molecules dictates that populations will proceed in the direction of ever greater vibrational excitation until the electrons return to their ground state. Here we only consider the isolated molecule situation.

When dealing with aromatic hydrocarbons, where spin-orbit coupling is small, it is convenient to discuss the problem in terms of zero-order pure spin states and small couplings between them. Thus for a closed-shell system we have a singlet manifold and a triplet manifold. Transitions between electronic states within a spin manifold are called internal conversion (IC), whereas transitions between spin manifolds are called intersystem crossing (ISC). The rates of IC and ISC in a classical kinetic framework are determined by Franck-Condon Factors (FCF's), spin-orbit coupling (SO), and Born-Oppenheimer 
operators (BO), as well as the gaps between electronic states $(\Delta)$, and the density of vibrational levels of the lower state isoenergetic with the optically pumped level of the upper state $(\rho) .^{2,3}$

Following the development of radiationless transition theories, pump-probe ionization experiments in supersonic beams enabled the measurements of the time-dependent populations of not only the singlet electronic states, but also the triplets. In a collisionless supersonic beam environment, ISC proceeds from the excited singlet state to a vibrationally excited triplet isoenergetic to the singlet. Therefore the triplet has a vibrational energy equal to the electronic energy difference between $\mathrm{S}_{1}$ and $\mathrm{T}_{1}$, and has no means of losing energy during the time scale of the experiment (phosphorescence is too slow). For benzene ${ }^{4,5}$ and a variety of substituted benzenes $s^{6,7,8}$ and related azines ${ }^{9,10}$, it was found that large-molecule kinetic mechanisms involving competing IC and ISC processes in isolated molecules fit the data quite well. The singlet ionization signals decay with the fluorescence lifetime, while the triplet signals build up during this period and subsequently decay exponentially (presumably by a second ISC to the singlet ground state) with lifetimes of around a microsecond. This was taken as validation of the general theoretical framework that had been developed.

More recently, studies have begun to appear which may indicate that perhaps the whole story has not been told about the excited state dynamics of larger molecules. First of all, a series of papers by Kato, Baba and coworkers ${ }^{11,12,13,14}$ described the use of ultra-high resolution CW Zeeman spectroscopy to measure the magnitude of SO coupling in the singlet excited states of various hydrocarbons. For each molecule without a heteroatom incorporated, they reported that they could not detect enough coupling to account for a finite ISC rate. Secondly, in our laboratory it was found that phenylacetylene and benzonitrile pumped by nanosecond lasers did not follow a classical kinetic scheme at all. ${ }^{15,16}$ Their triplet states were entirely populated during the laser pulse--only--and then the triplet populations did not decay at all during the $140 \mu$ s we could observe them in the beam. This is completely inexplicable in the generally accepted model of the way larger isolated molecules are supposed to behave. On a 
different time scale, Fielding and coworkers ${ }^{17,18}$ have found using femtosecond pulses that in benzene, ISC and IC proceeds with ultrafast rates, an unexpected result given the longer-time experiments.

In this paper we present new results on naphthalene ( 2 rings), anthracene ( 3 rings, $D_{2 h}$ ), and phenanthrene ( 3 rings, $C_{2 v}$ ) which demonstrate that unusual behavior is not restricted to benzene with triple-bonded substituents ${ }^{16}$, but is a general property of a larger group of hydrocarbons when examined by nanosecond pulsed lasers in a supersonic beam.

\section{Experiment}

The apparatus used for the recent experiments has been described previously. ${ }^{15,16}$ All were done in a pulsed, skimmed supersonic beam of helium expanding from about 3 atm, seeded with the molecule of interest. The pulsed valve and sample chamber immediately behind it were heated to temperatures between $60 \mathrm{C}$ and $150 \mathrm{C}$ to provide adequate vapor pressures of the larger molecules studied here. Temperatures were typically $60 \mathrm{C}$ for naphthalene, $150 \mathrm{C}$ for anthracene, and $130 \mathrm{C}$ for phenanthrene. The distance between the valve and the ion/electron collection point is $25 \mathrm{~cm}$.

Molecules seeded in the helium carrier gas were excited to their lowest singlet electronic state (usually the origin, but for naphthalene the $\overline{8}^{1}$ vibration at $32455 \mathrm{~cm}^{-1}$ was more often used because it is an order of magnitude more intense) by a YAG-pumped, doubled dye laser (about 8 ns pulse width), and subjected to a second pulse from a higher photon energy laser intended to probe the excited state population by ionizing only the excited molecules. The probe laser was either an argon fluoride excimer laser, or a YAG-pumped doubled or tripled dye laser, depending upon the wavelength desired. The two laser beams used were carefully combined to be coincident with dichroic mirrors, and counterpropagated together, unfocused, down the length of the supersonic beam. The timing between the lasers was controlled by programmable delay generators, with a timing jitter between 15 and 20 ns for the excimer/dye laser combination and $<10 \mathrm{~ns}$ when two dye lasers were used. The timing between the pulsed valve and the probe laser was kept constant and adjusted so the leading edge of the molecular 
pulse was always being sampled, avoiding excess clustering. Mechanical shutters in both laser beams allow for background subtraction during the course of data acquisition.

The results reported here depend upon two types of experiments--pump-probe time delay studies with ion detection, and pump-probe time-of-flight (TOF) photoelectron spectroscopy (PES) at various time delays. The basic experiment for examining the evolution of excited state populations is a time-delay scan. Ions created by the probe laser beam were sent into a TOF mass spectrometer, and only the parent mass peak was sampled. The time between the laser firings was varied from having the probe beam fired before the pump, to having the probe follow the pump by as much as $150 \mu \mathrm{s}$. Data is accumulated at each delay value for typically $2 \mathrm{~s}$ while cycling through the set of delays many times. This reduces the effects of long-time drift in the properties of the lasers. Laser intensities were set to minimize the amount of single-laser signal due to multiphoton ionization (which is fortunately minimal for these molecules because the $S_{1}$ energy is less than halfway to the ionization energy), but the shutters were used to subtract any residual direct ionization signal.

$193 \mathrm{~nm}$ TOF photoelectron spectra of the $S_{1}$ states of naphthalene and anthracene were recorded using a $30 \mathrm{~cm}$ flight tube, doubly shielded with mu-metal for magnetic isolation. No repeller voltage was used, and the electron flight times were recorded using a National Instruments transient digitizer card having a time resolution of $10 \mathrm{ns.} \mathrm{Again,} \mathrm{mechanical} \mathrm{shutters} \mathrm{were} \mathrm{used} \mathrm{for} \mathrm{single-laser}$ signal and background subtraction. As in time-delay scans, PES signals with various time delays were collected for short time periods and the delays were cycled through for long periods of time (up to two hours) so the spectra with different delays can be compared without being compromised by long term laser drift. The TOF data was converted to an energy scale using an $\mathrm{E}^{-3 / 2}$ factor (the Jacobian of the change from a time axis to an energy axis) to scale the intensities so that areas in the TOF and energy spectra are consistent. 
Intensity dependences of the singlet and triplet signals were obtained by varying the pump laser intensity while recording a time-delay scan at each setting. At higher intensities, the power was changed by using a variable wave plate and a polarizer, directly measuring the power at each point with a photodiode. For lower intensities, the power was further attenuated using a set of calibrated neutral density filters.

\section{RESULTS AND DISCUSSION}

A representative time delay scan is shown in Error! Reference source not found.. The signal shape looked the same for all wavelengths and molecules, consisting of a peak when the pump and probe lasers were overlapped in time, followed by an exponential decay to a constant value that persisted without attenuation as long as the flight time in our apparatus allowed--approximately $140 \mu$ s. The mathematical function for the signal (ignoring the influence of laser pulse shape) is therefore

$C=S_{1} e^{-\left(t-t_{0}\right) / \tau}+T ; t \geq t_{0}$

where $t_{0}$ is when the lasers are overlapped in time, and $\tau$ is the lifetime (determined by fluorescence) of the pumped singlet level. As will be explained in more detail later, $S_{1}$ is proportional to the population of the pumped singlet immediately following the pump pulse, and $T$ is proportional to the population of a long-lived triplet state formed during the pump laser pulse. The values of $S_{1}$ and $T$ were determined from each scan by convoluting the above function with a Gaussian representing the time widths of the lasers and any time jitter, then fitting the data to that function using a non-linear least-squares routine. It should be noted that there is no provision in this equation for any triplet population to be created after the laser pulse, unlike the case for benzene and various substituted benzenes studied earlier.

An ionization threshold curve for the triplet component $T$ can give considerable information about the character of the state. In order to produce the range of ultraviolet wavelengths for the probe laser necessary for this task, a variety of lasers, dyes and harmonic schemes had to be used. Since it is 
not possible to maintain consistent control of the intensities and beam qualities over this variety of methods, it was decided to use the ratio $T /\left(T+S_{1}\right)$ as the measure of the $T$ ionization probability. This ratio is much less sensitive to intensities and beam quality than a measure of $T$ alone, and as shown later in kinetic analyses, it is almost insensitive to laser intensity. It has the disadvantage that it depends upon both the singlet and triplet ionization cross-sections as a function of the probe wavelength, but the probe photon energy at the triplet ionization threshold excites a singlet, $\mathrm{S}_{1}$, molecule to considerably higher than its threshold, so in general it can be expected the $\mathrm{S}_{1}$ ionization curve will be continuous and fairly flat over a small range. Some checks on this determined it to be true in the regions of interest. Therefore ionization ratios were measured as a function of wavelength by doing time delay scans approximately every nanometer of the probe laser from the triplet ionization threshold to $198 \mathrm{~nm}$ using doubling and tripling of dye lasers in BBO, and at 193nm using an ArF excimer laser. These ratios were repeatable between different dye lasers, different harmonic schemes, and from day to day.

Time delay scans for all the molecules in this study were done using the $193 \mathrm{~nm}$ laser for the probe photons. The triplet ionization ratios at this wavelength are given in table 1 for a group of aromatic molecules (for benzene, the only molecule on the list that follows a classical kinetic scheme for its radiationless transitions, the ratio given is $Q_{i s c} /\left(Q_{i s c}+Q_{f f}\right)$, where the $Q$ 's are the quantum yields for intersystem crossing and fluorescence $\left.{ }^{5}\right) .193 \mathrm{~nm}$ light is far enough above the ionization potentials of the triplet states of all these molecules that the ionization probability of both singlets and triplets should be substantial. The ionization ratios vary from zero to almost 0.9 , thus showing that the amount of triplet formed depends in some intricate way on the excited state structure of the individual molecule.

Figures 2 and 3 show the ionization ratio curves with respect to wavelength for naphthalene and anthracene, with the ionization threshold of the vibrationless $T_{1}$ state indicated (as determined by known state energies). Although it is expected that the ionization threshold of a vibrationally excited 
state would be quite blue shifted due to Franck-Condon factors, it is seen that for both of these molecules the ionization threshold is very sharp--much more abrupt than one would expect for a state with $10625 \mathrm{~cm}^{-1}$ or $12613 \mathrm{~cm}^{-1}$ of vibrational energy. It is also notable that the first major threshold step is exactly at the $D_{0}-T_{1}$ energy difference, and for anthracene there is almost no signal below that value, confirming that the long-lived state observed is $T_{1}$ in these molecules. There is a large and unexpected dip in the ionization curve of naphthalene centered at about $210 \mathrm{~nm}\left(47600 \mathrm{~cm}^{-1}\right)$. Interestingly, a very strong valence absorption feature appears in the calculated triplet-triplet absorption spectrum at that wavelength (see Figure 5 below). A possibility is that this valence absorption, along with internal conversion, is competing with the ionization continuum at those wavelengths and suppressing the ionization signal.

Error! Reference source not found. shows the photoelectron spectra of the $S_{1}$ ionization process at various delays between the pump and probe lasers for naphthalene. The higher energy signal resulting from $S_{1}$ ionization decays with the fluorescence lifetimes (300ns), while the lower energy electrons from triplet ionization are there from the beginning and do not decay with time. Like phenylacetylene, the naphthalene triplet signal does not increase during the singlet lifetime, indicating there is no ISC in the absence of the laser field. There is more overlap between the singlet and triplet photoelectron spectra than there is in phenylacetylene ${ }^{15}$ so the fact that all triplet signal is created during the laser pulse is less obvious. However, for all electron energies the signal can be fit to equation 1 , showing there is no intersystem crossing from the post-laser singlet population. For anthracene the fluorescence lifetime is comparable to the width of the laser pulse. That, and time jitter effects, prevent an estimate of the magnitude of radiationless ISC in that molecule, as does the small amount of $\mathrm{S}_{1}$ produced in phenanthrene. 
The results presented here parallel the behavior seen previously in phenylacetylene ${ }^{15,16}$, showing that molecule is not an exception, but part of a substantial group of larger molecules exhibiting similar excited state dynamics. The three major observations that are difficult to rationalize using current ideas about radiationless transitions in larger molecules are:

1) Triplet formation only takes place during the light pulse (at least for naphthalene).

2) The triplet population does not decay significantly over hundreds of microseconds.

3) Ionization thresholds for the triplet population are very sharp, and exactly at the ionization energy of a vibrationless level of $T_{1}$, even though the triplet molecules must have large amounts of vibrational energy.

\section{Triplet Formation}

In a normal molecule such as benzene (if that is indeed normal), triplet population is generated by intersystem crossing (ISC) from a singlet population and continues as long as the latter exists. The ISC rate is determined by the magnitude of spin-orbit ( $\mathrm{SO}$ ) coupling in the $\mathrm{S}_{1}$ state, and the density of states in the triplet manifold isoenergetic to $S_{1}$. Attempts have been made to measure the magnitude of the SO coupling in the molecules studied here by using ultra-high-resolution Zeeman spectroscopy ${ }^{11-14}$, but the results show that there is not enough mixing between the singlets and triplets in these molecules to account for any amount of ISC at all, much less the almost complete transfer seen here in phenanthrene (Table 1). In previous publications ${ }^{15,16}$ we have considered a variety of mechanisms for triplet production (such as photon emission of various sorts), but have failed to find any evidence for their existence.

Since we now know the behavior seen in PA is general, here we would like to propose a mechanism that has the possibility of explaining all the current observations--light-induced SO coupling, 
in which the singlet-triplet coupling takes place at the 2-photon level but population is driven down to the one-photon triplet level by stimulated emission.

An interesting aspect of all experiments done with pulsed lasers in a given experimental apparatus is that they are all done with about the same rate of population of the initial state. This is because the laser is turned up until one sees a signal, and then one stops increasing the power because multiphoton processes and detector saturation can compromise the measurement value. When the absorption cross-section of the initial transition is very small, a substantial amount of laser power must frequently be used.

Given that significant laser flux is used in the experiments, one must consider whether multiple photon processes are important in the photodynamics. In the simplest cases, the curves of a multiple photon process can be identified and described by the relationship $C=\sigma /{ }^{n}$, where $C$ is some concentration produced by light with an intensity I, absorbed with a cross-section $\sigma$. For a multiphoton transition with no resonance intermediates, $n$ is the number of photons involved in the transition, and is the slope of a plot of $\log C$ versus log I. However, even for transitions without intermediate resonances, this simple picture ignores depletion of the initial state and geometric effects related to saturation, so it is not often seen experimentally ${ }^{19}$. For the experiments discussed here, the kinetics of excitation are better described by sequential one-photon steps, in which case a simple exponential relationship between the overall transition probability and intensity is valid only as a limiting case. Also, since stimulated emission must also be considered, any fully resonant multiple photon process without dissipative branches is a classical example of coupled equilibria. Once equilibrium is established, a steady state is achieved, and the intensity dependence can approach zero, indicative of saturation. If the laser pulse is weak enough or short enough that equilibrium is not achieved, or if dissipative processes occur, the intensity dependence can be anything from zero to the number of photons involved. However in general, a log- 
log power dependence plot has significant curvature, so an intensity dependence can only be defined over a small intensity region. A case that becomes fairly linear over large intensity spans for a two-step resonant process is one with a small initial cross-section and a much larger intermediate-to-final-state cross-section. If there is a substantial loss mechanism at the 2-photon level the intensity dependence of the intermediate state population can become one or less, since the initial transition would be the ratelimiting step, even though it is a 2-photon process.

SO coupling in aromatics has been studied previously in the context of accounting for the long phosphorescence lifetimes in condensed phases. Marian ${ }^{20}$ has recently reviewed the state of theoretical calculations of SO for large molecules and its role in ISC. Before modern electronic structure calculations were available, Henry and Siebrand ${ }^{21}$ made the argument that the primary sources of the magnitude of the $T_{1}-S_{0}$ transition moment were $\pi-\sigma^{*}$ and $\sigma-\pi^{*}$ states coupled into $T_{1}$ by SO perturbation. More recently, Agrens et $a l^{22}$ confirmed this using the multiconfigurational quadratic response method. It has also been a common theme that molecular distortions from vibrational motion are a potent source of SO coupling and its effect on ISC. ${ }^{23}$

Thus it is generally accepted that, although $\pi-\pi^{*}$ states of hydrocarbons without heteroatoms usually have very small SO coupling, $\pi-\sigma^{*}, \sigma-\pi^{*}$ and $\sigma-\sigma^{*}$ states have substantially more. These latter states are expected to be present at the two photon energy level, so mechanisms involving higher excited states must be considered.

The discussion immediately above is particularly relevant here because a common characteristic of all the molecules considered here is that the $S_{1}-S_{0}$ transitions have very low oscillator strengths, almost negligible in comparison to those of higher electronic states. Once an $\mathrm{S}_{1}$ population is created, one must then consider the possibility of transitions to states one photon higher in energy than $\mathrm{S}_{1}$, and these transitions have oscillator strengths that may be orders of magnitude larger than the initial one. If 
that is the case, the molecules will rapidly absorb another photon, resulting in a mixture of states at the two photon level. These will be strongly coupled to each other and to isoenergetic states in the triplet manifold because of the strong SO coupling at that level. Once populated, those mixed states can undergo stimulated emission down to the lower triplet levels, in effect creating the equivalent of an ISC process. Whether that up-down process through the two-photon level is viable for a given molecule will depend upon the cross-sections for the $S_{1}$ absorption and the $T_{n}$ stimulated emission probability (which is equal to the absorption cross-section of the lower triplet at the appropriate geometry). This information is not available experimentally, but can be obtained from electronic structure calculations.

\section{Calculated Excited State Absorption Spectra}

To examine the proposition that optical coupling between excited states is a viable mechanism for inducing intersystem crossing, we have calculated the excited state compositions and oscillator strengths for states that are optically coupled in one photon to the $S_{0}, S_{1}, T_{1}$ and $T_{2}$ states of phenylacetylene, benzonitrile, naphthalene, anthracene and phenanthrene. For this we used the SAC-CI package in Gaussian (equivalent to the Equations of Motion (EOM) coupled-cluster method found in other electronic structure calculation collections) with an augmented, correlation-corrected, double zeta basis set (aug-cc-pVDZ), at level one, and with 25 excited states of each allowed symmetry. As many as $12 \sigma$ orbitals are found within the energy range of the $\pi$ orbitals of naphthalene, for example, so $\sigma$ involvement in transitions of modest energy are to be anticipated.

To provide a graphical representation of these results, simulated $S_{1}$ and $T_{n}$ absorption spectra are given in Figure 5. There the oscillator strengths are convoluted with a Gaussian band shape of 800 $\mathrm{cm}^{-1}$ width (chosen because of the experimental width of the $S_{3}-S_{0}$ band in the naphthalene absorption spectrum of George and Morris ${ }^{24}$, but much smaller than the probable width of any valence state at the $60,000 \mathrm{~cm}^{-1}$ level) and summed to give a spectrum scaled as a molar extinction coefficient $\varepsilon$ that can be 
compared to experiment. In $D_{2 h}$, symmetry labels change with the choice of axis system and here they follow the Mulliken convention, where the long axis of the molecule is $y$, the in-plane short axis is $z$, and out-of-plane is $\mathrm{x}$. While these simulations are not expected to be identical to an experimental absorption spectra (high Rydberg and continuum orbitals are not included in the basis set, and the degrees of lifetime broadening of higher states are not known), they provide a basis for discussing a proposed mechanism.

Some experimental ultraviolet and vacuum ultraviolet spectra ${ }^{24,25,26}$ are available to assess the quality of the calculated spectra from the ground state of each molecule, and also some triplet-triplet absorption spectra, over small energy ranges. The latter were obtained from molecules contained in low-temperature solid solutions.

The vacuum UV spectrum of each molecule consists of a series of broad, intense, valence absorptions superimposed by sharper and weaker Rydberg lines. The broad structure (as well as the near-UV spectrum) is remarkably well reproduced by the SAC-Cl calculations, with relative intensities close to experiment. The calculated $S_{0}$ VUV spectrum of naphthalene is shown in Error! Reference source not found., compared with an experimental spectrum adapted from Koch, Otto and Radler ${ }^{25}$. Intensities are scaled to make the highest peaks equal since the experimental spectrum does not have a quantitative amplitude scale. It is seen that the major peak is coincident in energy, while the higher calculated energies seem to be low by about $0.2 \mathrm{eV}$. For anthracene, all apparent peaks are reproduced except for one at $7.0 \mathrm{eV}$, which is calculated at $7.3 \mathrm{eV}$ (and relative intensities are still good). For phenanthrene, all experimental valence peaks are reproduced within $0.1 \mathrm{eV}$ by the calculations.

The triplet-triplet experimental spectra (available for naphthalene ${ }^{27,28}$, anthracene ${ }^{27,28,29}$, and phenanthrene ${ }^{28}$ ) usually consist of a series of lines which we take to be a vibrational progression of around $1450 \mathrm{~cm}^{-1}$, of a single state, at an energy below the $S_{1}$ transition energy. One can estimate a 
solvent shift by comparing a solution UV spectrum with the corresponding vapor spectrum of each molecule. For naphthalene and phenanthrene the shifted calculated spectrum has a strong peak within $500 \mathrm{~cm}^{-1}$ of the experimental spectrum. For anthracene there is a $2500 \mathrm{~cm}^{-1}$ discrepancy.

In addition to transition rates, the SAC-Cl calculations provide a quantitative measure of configuration mixing, giving an indication of the amount of $\sigma-\pi$ mixing at the two photon level. Examination of the orbital content of each state of naphthalene showed that the ${ }^{1} \mathrm{~B}_{2 \mathrm{~g}}$ states (out-ofplane polarized from the one-photon levels) are all $\pi-\sigma^{*}$ and $\sigma-\pi^{*}$ as expected. However they have very little oscillator strength. The strongest transitions from $S_{1}$ are to $A_{g}$ (long axis) and $B_{1 g}$ (short axis) states. On examining orbital content, it is found that there is a substantial contribution from $\sigma-\sigma^{*}$ excitations for some of the states of these symmetries. Indeed, in the 30,000 to $40,000 \mathrm{~cm}^{-1}$ range of transition energies from $S_{1}$, the states with high oscillator strength have $\sigma-\sigma^{*}$ character in the range of $10-14 \%$. A strong SO coupling is therefore likely, based on the conclusions of Henry and Siebrand ${ }^{21}$.

\section{Optical transitions to and from the higher excited states of large molecules}

For transitions to the $S_{1}$ states of large molecules, both the initial and final states can be considered to be basically stationary, and described well by the usual Born-Oppenheimer (BO) wavefunctions. On progressing to states that have twice the $S_{1}$ energy, the density of both electronic and vibrational states at that level makes the situation much more complicated. It is important to realize that the density of electronic states at the 2-photon level is very large for these molecules. For example, for phenanthrene there are 56 total calculated singlet and triplet states between 7 and $8 \mathrm{eV}$ (or 110 if you count the triplet spin sublevels separately). That means the average electronic state spacing is less than the zero point energy of most vibrations, and one could consider this energy range to be a continuous distribution of conical intersections. A rigorous treatment of the evolution of a state at the 2-photon therefore presents a significant challenge. As illustrated by the continuous nature of 
the optical spectrum, each valence state of a given energy is broadened and mixed with all other states near to that energy to one extent or another by BO breakdown and other perturbations such as SO coupling. However, the upper state of any optical transition to the two-photon level will be a nonstationary superposition state consisting only of optically allowed components that will begin to evolve on the timescale of both electronic and nuclear motions. This non-stationary state can be written as a sum of BO states:

$\Psi_{\mathrm{f}}=\sum_{\mathrm{k}, \mathrm{j}} \mathrm{c}_{\mathrm{kj}}(t) \psi_{k} \chi_{j}\left(\boldsymbol{R}_{0}^{k}, Q_{j}^{k}\right) e^{-i \omega_{k j} t}$

$\omega_{\mathrm{kj}}=\omega_{\mathrm{k}}+\omega_{\mathrm{j}}$, where $\omega_{\mathrm{k}}$ is the electronic frequency and $\omega_{\mathrm{j}}$ is the vibrational frequency; $\psi_{k}$ is the electronic wavefunction; $\chi_{\mathrm{j}}$ is the $\mathrm{j}^{\text {th }}$ vibrational wavefunction of state $\mathrm{k}$, with an equilibrium nuclear geometry of $\boldsymbol{R}_{0}^{k}$, and normal coordinates $\left\{Q_{j}^{k}\right\}$; and when the molecule is in a radiation field, to first order:

$c_{k j}(t)=-\frac{e}{m} \int_{0}^{t}\left\langle\psi_{k}|\boldsymbol{A} \cdot \nabla| \psi_{i}\right\rangle\left\langle\chi_{j} \mid \chi_{i}\right\rangle e^{i \omega_{k i} t^{\prime}} d t^{\prime}$

$\psi_{i}$ and $\chi_{i}$ are the electronic and vibrational wavefunctions of the initial state, and $\omega_{\mathrm{ki}}=\omega_{\mathrm{kj}}-\omega_{\mathrm{i}}$, while $\boldsymbol{A}$ is the vector potential of the radiation.

It is seen that having a finite $c_{k j}(t)$ depends upon having a finite oscillator strength and FranckCondon factor, so the radiation field prepares an initial higher state only using components of the final eigenstate with these characteristics. Following the initial preparation, the superposition state will dephase and evolve at the whims of small intramolecular perturbations and the changed interaction potential between the electrons and nuclei. Due to the localized nature of the initial vibrational wavefunction, the initial superposition state will have the same geometry as the lower state, but the equilibrium geometry of $\Psi_{f}$ will not be the same as $\psi_{0}$ and the molecule can travel away from that location in nuclear space on a short timescale, mixing in other electronic states as it goes. 
When each level is resonant in a multiple photon process, the steps can be considered as a sequential linear rate scheme. However, unless there is a very rapid dissipative process in some upper level, such as direct dissociation, one must also consider stimulated emission downward from each photon level, since the rate of a backward transition is equal to the forward rate. Particularly when an upper state is highly mixed, all lower states are in play for a downward destination that meet the energy requirements, not just the states visited on the way up. Therefore, components of the superposition state that did not carry oscillator strength from the set of upward transitions can initiate transitions downward to states at fewer-photon levels that cannot be populated directly from the ground state.

To make the process more definite for the present purposes, it is convenient to introduce a classical rate scheme that can be used to examine the consequences of the various transition rates in the molecules considered here. A plausible scheme of minimal complexity is shown in Error! Reference source not found.. Following a transition to $S_{1}$ with a cross-section $\sigma_{1}$, the molecule rapidly establishes another equilibrium with a nonstationary singlet state at the two-photon level with cross-section $\sigma_{2}$, as well as having the possibility of undergoing ISC to the triplet state at the one photon level with a rate $k_{2}$ and fluorescing with a rate $k_{1}$. Meanwhile, some components of the singlet two-photon superposition state can establish a different equilibrium (ISC) to the triplet manifold with a rate $k_{3}$. There are dissipation channels to a sink in the singlet manifold $\left(k_{5}\right)$ that allows for losses by internal conversion at the 2-photon level, and also by ionization (with a cross-section $\sigma_{3}$ ). In the triplet manifold the one- and 2-photon levels are connected by a cross-section $\sigma_{4}$, and an initial state populated at the one-photon level is connected to a relaxed state by internal relaxation or IC $\left(k_{4}\right)$. All cross-sections can be considered to be first order in the laser intensity, and therefore the rate is represented by the product of a cross-section and a time-dependent intensity, $k_{n}=\sigma_{n} l(t)$. Here we will assume the laser pulse has a Gaussian intensity dependence centered on a time $t_{0}$, giving $I(t)=e^{-\left(t-t_{0}\right)^{2} / \gamma^{2}}$, where $\gamma=0.926 \mathrm{~W}, \mathrm{~W}$ 
being the full width at half maximum of the pulse (a trial inclusion of a rapid oscillation superimposed on the Gaussian to simulate mode mixing in the unseeded YAG laser did not alter any results).

This scheme can be represented by a set of coupled rate equations:

$\frac{d A}{d t}=-e^{-\left(t-t_{0}\right)^{2} / \gamma^{2}} \sigma_{1}[A-B]+k_{1} B$

$\frac{d B}{d t}=e^{-\left(t-t_{0}\right)^{2} / \gamma^{2}}\left[\sigma_{1}(A-B)-\sigma_{2}(B-C)\right]-\left(k_{1}+k_{2}\right) B$

$\frac{d C}{d t}=e^{-\left(t-t_{0}\right)^{2} / \gamma^{2}}\left[\sigma_{2}(B-C)-\sigma_{3} C\right]-\left(k_{3}+k_{5}\right) C+k_{3} D$

$\frac{d D}{d t}=e^{-\left(t-t_{0}\right)^{2} / \gamma^{2}}\left[\sigma_{4}(E-D)\right]+k_{3} C-k_{3} D$

$\frac{d E}{d t}=e^{-\left(t-t_{0}\right)^{2} / \gamma^{2}}\left[\sigma_{4}(C-E)\right]-k_{4} E+k_{2} B$

$\frac{d G}{d t}=k_{4} E$

$\frac{d Z}{d t}=\sigma_{5} e^{-\left(t-t_{0}\right)^{2} / \gamma^{2}} C+k_{5} C$

where $A=\left[S_{0}\right], B=\left[S_{1}\right], C=$ a singlet superposition state at the 2 photon level, $D=$ a mixed triplet state at the 2 photon level, and $E=$ a triplet state at the one-photon level $\left(T_{1}\right.$ or $\left.T_{2}\right) . Z$ (not shown in Error! Reference source not found.) is a sink representative of products of IC and ionization from the 2-photon level. It was also found necessary to include another sink at the one-photon triplet level, state $G$, that represents irreversible relaxation there. $\mathrm{G}$ is the long-lived triplet state seen in the experiment. This equation set was numerically integrated (using the Runge-Kutta method) as a function of time, assuming all population is initially in $\mathrm{A}$, and the changing relative populations determined for all levels as a function of laser intensity. Initial estimations for the cross-sections were obtained from the SAC-CI calculations, and the magnitudes and trends in the population ratios compared to experimental results. 
One way of looking at the kinetic scheme described above is that, for the most part, it is a systematic method of introducing additional basis functions into the description of the electronic structure of a molecule being significantly polarized by a radiation field. Electrons respond much more rapidly to the light than the nuclei and the electronic states are driven into equilibrium before the nuclei. The various equilibria and relaxation processes at different levels of excitation then distribute the population into various electronic states at the end of the pulse, at which time internal interactions take over directing the state evolution. This makes the distribution of products intensity dependent in a very complicated way that is not directly correlated to the number of photons involved in the process. In this picture levels $C$ and $D$ are the pure singlet or triplet nonstationary components of a mixed dressed state with both singlet and triplet character, and the 2-photon ISC rate $k_{3}$ is the dephasing rate of the components. States C, D, and E can all be considered as components of the evolving, radiationpolarized wavefunction of the molecule during the course of the pulse, as represented by equation 2 . This description of the dynamics at the 2-photon level is undoubtedly simplistic, and is the weakest part of this kinetic model.

Transitions downward from the two-photon level into the lower triplet manifold can terminate in any electronic state whose potential minimum is below the one-photon energy. The SAC-CI calculations indicate that there are either two or three of those states at the ground state geometry for the molecules studied here. States just above the one photon energy at the $\mathrm{S}_{0}$ geometry could well drop below at a different geometry or when a zero-point correction is applied, so they could be considered to be lower state candidates also. For all of the molecules but anthracene, $T_{1}$ has a different symmetry than $S_{1}$, and there is a triplet state of $S_{1}$ symmetry just below the $S_{1}$ energy that can be reached from the 2-photon superposition state given reasonable Franck-Condon overlap. 
The energy gap between the lower triplet state energy and the $S_{1}$ energy must eventually appear in any $T_{1}$ product as vibrational energy for conservation purposes. If the downward transition happens before any IC can take place, the Franck-Condon factors (FCf's) for the transitions would favor lower triplet electronic states near to the $S_{1}$ energy. Alternately, considerable electronic energy can be transferred in the upper level to vibrations. In that case, since there are a very large number of states in these molecules just below the two photon level, given time, the molecule could start to cascade down through these electronic states, transferring energy to the nuclear motion while keeping the total energy in the molecule the same. This relaxation would inhibit the maintenance of an equilibrium with the singlet levels however, and is explicitly included as IC in the kinetic scheme listed above, only as a sink for population.

The above kinetic scheme can be used to predict the $S_{1}$ and $T_{1}$ populations at the end of the laser pulse, as a function of the intensity of the light, and these population-versus-intensity curves can be compared to measured signals.

Estimated rates from absorption spectra can serve as initial values for some cross-sections, and some rates are constrained by experimental observations. For example, the fact that no triplet is formed in naphthalene after the laser pulse, forces $k_{2}$ to be zero. Also, it can be anticipated that the 2photon IC, $k_{5}$, will be relatively large from general experience in resonance enhanced multiphoton ionization spectroscopy (REMPI) ${ }^{30,31}$. In that method, for larger molecules the only states seen as twophoton resonances are Rydberg states. ${ }^{32}$ This is because IC competes with ionization for valence resonances in the upper singlet manifold even though quite strong laser intensities are used in REMPI. For naphthalene, there does not seem to be any detectable REMPI spectrum at all in the $\mathrm{S}_{1}$ region below the 2-photon IP ${ }^{33}$, and there was negligible ionization seen from the pump laser in the present experiments, indicating $k_{5}$ is substantial. 
For naphthalene, the absorption calculations indicate that the ratio of oscillator strengths at the one- and two-photon levels is about 200. This, however, neglects vibronic effects in $\mathrm{S}_{1}$ and overlapping bands at the 2-photon level. Also the $\overline{8}^{1}$ vibrational band used for most measurements is a factor of 10 more intense than the origin, so a $\sigma_{2} / \sigma_{1}$ ratio of $20-100$ is a reasonable initial estimate. $\sigma_{4}$ is expected to be about double $\sigma_{2}$. Starting from those estimates, the fitted $S_{1}$ and $T_{1}$ intensity curves for naphthalene are shown in Error! Reference source not found.. The rate equation values used to reproduce the experimental curves are: $\sigma_{1}=4 \times 10^{7} ; \sigma_{2}=2.5 \times 10^{9} ; \sigma_{3}=0 ; \sigma_{4}=5 \times 10^{9} ; k_{2}=0 ; k_{3}=2 \times 10^{7}$; $k_{4}=2 \times 10^{8}$; and $k_{5}=2.9 \times 10^{8}$. Since the absolute values of the laser intensities are not known, their units in the program are scaled such that the values are exactly one at the maximum intensity, and the rates resulting from them have the values of the $\sigma$ 's at that point. This scheme has the additional advantage that all kinetic parameters and cross-sections have units of $\mathrm{s}^{-1}$ since the intensities are represented by dimensionless ratios. The above values are all quite reasonable numbers, but should not be considered definitive since they are quite dependent on the kinetic model, and may not even be a unique solution to the one used. The results show, however, that this simple kinetic scheme is capable of reproducing the experimental trends. Other, simpler schemes that were tried could not simultaneously reproduce the curvatures, the slopes and the magnitudes. Slopes on a log-log plot of the experimental data for naphthalene versus intensity gave power dependences of 0.67 for the singlet and 0.71 for the singlet, again emphasizing the important role of the IC at the two-photon level and the difference in the singlet cross-sections.

The most challenging feature in the intensity dependence curve to reproduce in the kinetics is the positive curvature of the triplet curve at low intensities. Since in a kinetic scheme the states are sequentially populated, if the triplet population has to go through the 2-photon level (versus crossing directly at the one-photon level) there is naturally a negative curvature of the triplet signal with intensity because of a quadratic intensity component. ISC at the one photon level is precluded for naphthalene 
because experimentally it is found there is no triplet formation after the laser pulse, which would happen if $k_{2}$ had any significant magnitude, given the long fluorescence lifetime. Together, the large $\sigma_{2}$ $/ \sigma_{1}$ ratio and IC in the 2-photon singlet redistribute the populations in such a way that a positive curvature is obtained.

For anthracene, even though the $\mathrm{S}_{0}-\mathrm{S}_{1}$ oscillator strength is much larger than in naphthalene, since a strong vibronic band was used in the latter, the $\mathrm{S}_{0}-\mathrm{S}_{1}$ cross-sections are comparable. The intensity curves are also quite similar, with an initial rise followed by a fairly linear increase for the triplet, and a somewhat curved increase for the singlet. Because of a very low power dependence in this molecule, it is possible to attenuate the pump laser considerably and still see signal, providing measurements over three orders of magnitude of intensity and making a log-log plot desirable, as in Error! Reference source not found.. There the singlet and triplet data points are accompanied by a linear least-squares fit to each logarithmic data set (black lines), and a fit from the kinetic equation calculations (dashed lines). The slopes of the least-squares lines are 0.47 for the singlet and 0.52 for the triplet.

The SAC calculated absorption spectra only show a modest singlet cross-section to the twophoton level, and indicate $\sigma_{1} \approx \sigma_{2}$. Due to large uncertainties in the position of a single, large, calculated triplet-triplet absorption peak shown in Figure 5, it is difficult to estimate $\sigma_{4}$ from the simulated spectra. Also, for anthracene, the fluorescence lifetime is only a small amount larger $(\sim 18 \mathrm{~ns})^{34}$ than the laser pulse width, so it is not possible to estimate the value of $k_{2}$ from whether triplet is formed after the laser pulse. The additional flexibility in allowing a finite value of $k_{2}$ is useful, and a reasonable fit to the experimental data over the entire intensity range is obtained with the kinetic values: $\sigma_{1}=2 \times 10^{8}$; $\sigma_{2}=2 \times 10^{10} ; \sigma_{3}=0 ; \sigma_{4}=2 \times 10^{7} ; k_{2}=6 \times 10^{6} ; k_{3}=4 \times 10^{8} ; k_{4}=1 \times 10^{9} ;$ and $k_{5}=1 \times 10^{9}$. With the exception of $k_{2}\left(\mathrm{~S}_{1}\right.$ ISC), all the $k_{n}$ are similar to naphthalene. The very small amount of one-photon ISC (only about 1/10th 
of the fluorescence rate) is needed to provide enough triplet population at low intensities. At higher intensities the triplet population is provided by the 2-photon ISC $\left(k_{3}\right)$, which is much larger than $k_{2}$, and the upper parts of the curves can be reproduced well without using $k_{2}$. The fraction of population going into IC from the 2-photon level varies considerably over the intensity range, going from 0.01 at the lowest power to 0.95 at the highest. It is primarily this sink that creates the very low power dependence exponent. Also, it is seen that the $\sigma_{1}$ and $\sigma_{2}$ values emerging from the fit are not what one would expect from the calculated absorption spectra (i.e. not about equal). This may be due to deficiencies in the electronic structure calculations, in the kinetic scheme, or both, and needs to be examined further. However, since the experimental log-log plot is surprisingly linear over such a large intensity range, indicative of a rate-limiting step, an error in the SAC-calculated $\sigma_{2} / \sigma_{1}$ ratio is suspected. This is most likely due to the fact that calculations were only done in $D_{2 h}$ symmetry, which eliminates $S_{1}-S_{2}$ vibronic mixing that would introduce the same strong singlet transitions seen in naphthalene. Since a singlegeometry calculation uses two months of cpu time, a full vibronic calculation is not practical at the present time.

The number of adjustable parameters in the kinetic scheme is fairly large and it would seem that almost any data set should be able to be reproduced. This, however, is not the case because the ranges are highly constrained by experimental and theoretical considerations and, particularly for anthracene, the large number of experimental points over a large intensity range. It is quite challenging to find a set of parameters that achieve a satisfying reproduction of the experimental results over the entire intensity range, but many parameters are not completely linearly independent. Thus the values given should not be construed as proven rate measurements, but as a demonstration that a kinetic model can be viable. 
A comparison between phenylacetylene and benzonitrile further illustrates how the calculated absorption spectra can help to explain experimental results. The former has a substantial triplet ratio (with $193 \mathrm{~nm}$ ionization) of 0.25 , while the isoelectronic benzonitrile's ratio is only 0.04 . Looking at the absorption curves in figure 5 , it is seen that at the energy of the light used to pump the origin of $S_{1}$ is below all the strong absorptions of $S_{1}$ benzonitrile to the 2-photon level, while for phenylacetylene, the light energy is in the middle of several strong bands. There is also a noticeable similarity between the $\mathrm{S}_{1}$ and the $T_{2}$ absorption curves, not surprising since those two states have almost identical sets of configurations. Therefore whenever a superposition state gets formed at the 2-photon level it could immediately be stimulated down to the $T_{2}$ level without even changing its electronic structure. Only the spin needs to flip. The power dependence of the triplet signal of phenylacetylene was previously measured to be $1.12,{ }^{15}$ so in this small sample of molecules the power dependence scales inversely with the size of the molecule (and thus the density of electronic states at the 2-photon level).

A more sophisticated way to model the evolution of excited electronic states is the multistate density matrix approach ${ }^{35}$. This method can correctly take into account the coherences possible in a laser driven process, as is often necessary for atoms and small molecules. Most studies have indicated that for large molecules and complex kinetic processes, inherent relaxation and interference effects destroy any coherence before it can build up ${ }^{36,37}$, so results are the same as when using kinetic rate equations. However, for completeness, the above kinetic scheme was implemented using density matrices, with appropriate estimates of various relaxation rates. In agreement with previous indications, there was very little difference in the cross-sections and rates obtained in fitting the experimental data using density matrices versus using rate equations.

\section{Triplet State Properties}


For the molecules studied here some of the molecules end up in a highly vibrationally excited triplet state when the laser pulse ends. The vibrational energy in the triplet is not a thermal distribution, but a finite number of vibrational levels that are isoenergetic with the pumped $S_{1}$ level within the transform bandwidth of the molecular rovibronic state that is excited in a particular molecule. Using the method of Haarhoff ${ }^{38}$ (his equation 2), the experimental energy gaps, and calculated vibrational frequencies it is possible to calculate the density of triplet states per $\mathrm{cm}^{-1}$ at the $S_{1}$ level, as given in table 1. Using the experimental singlet lifetimes to calculate the transform bandwidth, we can determine the approximate number of triplet states that can couple to a single $S_{1}$ level for each molecule. For most of these molecules, $\Delta v_{\text {Rad }} \approx 1 \times 10^{-4} \mathrm{~cm}^{-1}$, so the number of coupled states ranges from about 10 for benzene to almost $10^{12}$ for anthracene. In spite of considerable discussion about an "energy gap" rule ${ }^{39}$, when comparing molecules for the purposes here, where the energy gaps are not dissimilar, the main determinant in the density of states is the number of atoms. That is because this number, in turn, determines the number of vibrational modes, which appears as the exponent of the energy gap in the Haarhoff formula. Thus phenylacetylene has about $4 \times 10^{5}$ coupled states after the laser pulse, explaining the difference between its behavior and that of benzene, which has only a few.

During the laser pulse, one must use the transform width of the laser to determine how many states are coupled. For an $8 \mathrm{~ns}$ laser pulse the transform width is about $6 \times 10^{-4} \mathrm{~cm}^{-1}$, so the number of coupled states will be somewhat larger than when using the radiative width, but for most of these molecules the difference is scarcely of importance. However for picosecond and femtosecond pulses the transform width encompasses many other rovibronic states and the picture changes considerably.

From table 1 we see that the total vibrational energies arising from the $S_{1}-T_{1}$ gap can be 7500 to $12500 \mathrm{~cm}^{-1}$, which means that the vibrational energy contents of triplet states isoenergetic to $\mathrm{S}_{1}$ are thousands of wavenumbers past the point where vibrational normal modes have any relevance. Beyond 
a few thousand wavenumbers of excitation the anharmonic coupling between modes makes it is necessary to go over to a local mode picture of the vibrational motions. ${ }^{40}$ At high vibrational excitation, one must consider many different local modes excited, all with different phases, so the atoms are moving around in almost a random fashion with respect to one another. And at the energies considered for the present molecules, there will be $10^{5}$ to $10^{12}$ of those modes, all within an energy range of a few hundredths of a wavenumber.

When considering the wavefunction of a molecule it is common to make the Born-Oppenheimer (BO) approximation, where the electronic and vibrational parts of the wavefunction can be considered to be separable. The physical justification of the BO approximation is that the electrons, being much lighter than the nuclei, can adiabatically follow the motion of the nuclei, and the nuclear coordinates can be considered as a fixed parameter in the electronic wave functions at a given nuclear geometry (the adiabatic approximation). There are situations where the $\mathrm{BO}$ approximation is not valid, such as for regions of nuclear coordinate space where electronic potential energy surfaces are close to one another. In most systems, terms of the Hamiltonian ignored in the $\mathrm{BO}$ approximation are those responsible for the transfer of energy between electronic and vibrational motion described by BO wavefunctions.

In other situations, this adiabatic approximation becomes irrelevant. For Rydberg states with large principal quantum numbers the Rydberg electron is going slowly with respect to the nuclear motion, and is at such a large distance that the moving nuclei and core electrons are indistinguishable from a point charge. Then the Rydberg electron orbits do not depend at all on the pattern of nuclear motion, and behave entirely like atomic orbitals. We would like to suggest that another situation where the adiabatic approximation is irrelevant is in the highly excited vibrational states of large triplet molecules populated by ISC. 
In a superposition vibrational state with $10^{10}$ components and $10000 \mathrm{~cm}^{-1}$ of energy, the nuclei are moving rapidly and randomly with respect to one another. Any electron in a region of space sufficiently removed from any atom core that it sees the electric potential of several nuclei simultaneously would be subject to a rapidly varying and randomly changing potential. The electron would essentially decouple its motion from the nuclei, forming a wavefunction with respect to the average positions, in the same way a pendulum weakly coupled to a forest of other pendula oscillating with different phases and frequencies would move independently. The unpaired electrons for the $T_{1}$ states of the molecules discussed here are in pi orbitals that have no density in the plane of the nuclei, and have exposure to a number of nuclei at each point the wavefunction has significant amplitude.

The justification for this supposition is that it would shed light on the unexplained behaviors of the triplet states of these larger molecules--that the states have very long lifetimes and that their ionization thresholds are sharp. If the unpaired electrons in the triplet states were decoupled from the nuclei, there would not be enough interaction between them to enable ISC to the electronic ground state. Also, if the electron is mostly ignoring the nuclei then FCf's would be diagonal, and there would be an abrupt ionization threshold, as observed.

\section{CONCLUDING REMARKS}

Given the disparity between the $S_{0}-S_{1}$ and the $S_{1}-S_{2}$ absorption cross-sections in naphthalene and phenanthrene, it would be surprising if states at the 2-photon level were not involved in ISC. The conclusion that ISC at the one-photon level is either absent or small in naphthalene agrees well with the observations of Baba and coworkers ${ }^{11-14}$ that ISC is negligible. In their work CW lasers were used, so higher order processes are improbable, and at the one-photon level it is likely that indeed no triplet formation happens. The small amount of SO coupling arising from the kinetic model for anthracene may not be enough to show a significant Zeeman effect from the triplet, but that will have to be examined 
further. However, there is still a considerable conflict between their conclusion from Zeeman work that benzene has no $S_{1}$ singlet-triplet coupling ${ }^{41}$, and the experimental pulsed laser results ${ }^{15}$ that show a definite ISC after the laser pulse. One would have to conclude that there must be an additional effect that makes a CW Zeeman measurement different than a nanosecond pulsed experiment.

An interesting question is how much this understanding will contribute to interpreting the behavior of large molecules when excited with shorter picosecond and femtosecond pulses. Although the total photon flux in a given ultrafast pulse is less than in a nanosecond pulse, the peak powers of the lasers used in ultrafast experiments are orders of magnitude higher. Therefore, multiphoton process would be expected to be prevalent. Studies by Weber and coworkers ${ }^{42}$ found that in phenol and naphthalene, femtosecond pulses efficiently created states at the 2-photon level (which they called "superexcited" states) that they were able to study by time-delayed photoelectron spectroscopy. Clear signatures of high Rydberg states were seen, but there was also a broad background that may be due to valence components of a highly mixed state. The work by Fielding and coworkers ${ }^{17,18}$ on benzene reports an ultrafast ISC rate in the "channel three" upper vibrational region of $\mathrm{S}_{1}$. This is in contrast to the very slow (microsecond) ISC rates seen in our previous work on lower vibrations of $\mathrm{S}_{1}$ benzene5, ${ }^{15}$. In their experiment, the excitation energy is resonant with the upper part of the Franck-Condon envelope for the $S_{1}$ state, a region of very weak absorption. Thus it is possible that the 2 nd photon absorption cross-section is considerably higher than the initial one, and the linear intensity dependence seen is due to a rate-limited kinetic process. Rapid ISC at the 2-photon level is easier to rationalize, but if the reported rapid relaxation represents the 2-photon dephasing rate, the femtosecond pulses do not last long enough to stimulate the molecule down into the lower triplet states. Thus the low energy of the electrons in the photoelectron spectra cannot be easily explained when invoking a higher energy ISC. On the other hand, if a dominant part of a two-photon state consists of two-electron-excited configurations, low energy photoelectrons could occur because the primary ionization transitions would 
be to electronically excited cations. It would be of interest to explore this further. Ultrafast experiments on the polycyclic aromatics could well be instrumental in further elucidating the roll of higher states in the dynamic process in molecules following pulsed laser excitation.

Because of their large polarizabilities and relatively weak $S_{1}$ transitions, phenylacetylene and the polycyclic aromatics may represent an extreme in the range of molecular behaviors. However, there are a great number of molecules in this category, and for these the disregard of multiphoton processes is ill advised. Due to the complexity of the kinetic process, a simple power dependence measurement is insufficient evidence that higher states are not involved in the evolution of populations when pulsed lasers are used. We have not been concerned with the situation where very low powers are used, such as in ultrahigh resolution studies where CW lasers are necessary. However, even though short singlet lifetimes make 2-photon excitation in the singlet unlikely at low intensity, that does not mean multiple photon effects are impossible. If there is ISC at the one-photon level, a long-lived triplet could well be pumped back into the singlet, thus decreasing the triplet population from what one would predict from a given ISC rate.

Ultimately for larger aromatics, it seems likely that the comfortable picture of excited state dynamics between pure-spin $\mathrm{BO}$ states will have to be abandoned if the details of the process are to be understood. Only $\mathrm{S}_{0}$ and $\mathrm{S}_{1}$ are in that category, and better descriptions of all other states in a radiation field will possibly involve something like a density functional approach. The idea would be that there is a dressed state of the molecule characterized by a time-dependent electron density, which is connected to various irreversible pathways that lead to measurable product state populations. Hopefully this will also enhance the intuition that enables generality and insight.

\section{ACKNOWLEDGMENTS}


We gratefully valuable discussions with Prof. Thomas Weinacht concerning the dynamics of molecular excited states. TJS is supported at Brookhaven National Laboratory under Contract Nos. DE-AC0298CH10886 and DE-SC00012704 with the U.S. Department of Energy and supported by its Office of Basic Energy Sciences, Division of Chemical Sciences, Geosciences, and Biosciences. 
Table 1

Some properties of the molecules discussed in this work. All but benzene exhibit light-induced ISC. All energies are in wavenumbers, and the densities of states are in inverse wavenumbers.

\begin{tabular}{|l|l|l|l|l|l|l|l|l|}
\hline Molecule & $\begin{array}{l}\mathrm{S}_{1} \\
\text { Energy }\end{array}$ & $\begin{array}{l}\mathrm{T}_{1} \\
\text { Energy }\end{array}$ & $\begin{array}{l}\text { Ionization } \\
\text { Energy }\end{array}$ & $\begin{array}{l}\mathrm{S}_{1}-\mathrm{T}_{1} \\
\text { Gap }\end{array}$ & $\begin{array}{l}\text { Triplet } \\
\text { Ratio- } \\
193 \mathrm{~nm} \\
\text { Probe }\end{array}$ & $\begin{array}{l}\text { Density } \\
\text { Of } \mathrm{T}_{1} \\
\text { States at } \\
\mathrm{S}_{1}\end{array}$ & $\begin{array}{l}\mathrm{S}_{1} \text { Expt. } \\
\text { Osc. } \\
\text { Str. }\end{array}$ & $\begin{array}{l}\mathrm{S}_{1} \text { Theo. } \\
\text { Osc. Str. }\end{array}$ \\
\hline Benzene & $38086^{\mathrm{a}}$ & $29640^{\mathrm{b}}$ & $74555^{\mathrm{c}}$ & 8446 & $0.74^{\mathrm{d}}$ & $1.01 \times 10^{5}$ & $0.0001^{\mathrm{e}}$ & 0 \\
\hline Phenylacetylene & $35877^{\mathrm{f}}$ & $25400^{\mathrm{g}}$ & $71127^{\mathrm{h}}$ & 10477 & $0.25 \mathrm{f}$ & $3.9 \times 10^{9}$ & $0.0005^{\mathrm{i}}$ & 0.0003 \\
\hline Benzonitrile & $36513 \mathrm{f}$ & $26780^{\mathrm{j}}$ & $78490^{\mathrm{k}}$ & 9733 & 0.04 & $6.9 \times 10^{8}$ & $0.005^{\mathrm{l}}$ & 0.0030 \\
\hline Naphthalene-origin & $32019^{\mathrm{m}}$ & $21394^{\mathrm{n}}$ & $65687 \mathrm{~m}$ & 10625 & 0.34 & $4.5 \times 10^{10}$ & $0.002^{\circ}$ & 0.0004 \\
\hline Naphthalene-8 & 32455 & & & 11061 & 0.22 & $8.4 \times 10^{10}$ & & \\
\hline Anthracene & $27688^{\mathrm{p}}$ & $15075^{\mathrm{q}}$ & $59872^{\mathrm{r}}$ & 12613 & 0.43 & $6.9 \times 10^{15}$ & $0.018^{\mathrm{s}}$ & 0.0765 \\
\hline Phenanthrene & $29328^{\mathrm{t}}$ & $21780^{\mathrm{u}}$ & $63654^{\mathrm{v}}$ & 7548 & 0.88 & $2.1 \times 10^{12}$ & $0.003 \mathrm{t}$ & 0.0013 \\
\hline
\end{tabular}

${ }^{a}$ A. Sur, J. Knee and P. Johnson, J. Chem. Phys. 77,654-668 (1982).

b S. Sharpe and P. Johnson, J. Chem. Phys. 81, 4176-4177 (1984).

c L. Chewter, K. Muller-Dethlefs and E. Schlag, Chem. Phys. Lett. 135, 219-222 (1987).

${ }^{d}$ From $Q_{I s c} /\left(Q_{f l}+Q_{I s c}\right) ;$ C. Otis, J. Knee, and P. Johnson, J. Phys. Chem. 87, 2232-2239 (1983).

e M. Goeppert-Mayer and A. Sklar, J. Chem. Phys. 6, 645 (1938).

f J. Hofstein, H. Xu, T. Sears and P. Johnson, J. Phys. Chem. A 112, 1195-1201 (2008).

g EELS solid; P. Swiderek, B. Gootz and H. Winterling, Chem. Phys. Lett. 285, 246 (1998).

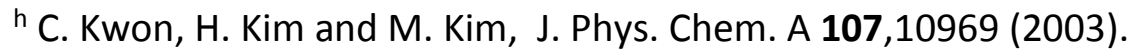

i G. W. King and S. P. So, J. Mol. Struct. 37, 543 (1971).

j K. Takei and Y. Kanda, Spectrochim. Acta 18, 1201-1216 (1962).

${ }^{k}$ M. Aarak, S. Sato and K. Kimura, J. Phys. Chem. 100, 10542 (1996).

I T. Peacock and P. Wilkinson, Proc. Phys. Soc. 79, 105 (1962).

m M. Cockett, H. Ozeki, K. Okuyama and K. Kimura, J. Chem. Phys. 98, 7763-7771 (1993).

${ }^{n}$ H. Gatterman and M. Stockburger, J. Chem. Phys. 63, 4541-4545 (1975).

${ }^{\circ}$ G. George and G. Morris, J. Mol. Spect. 26, 67-71 (1968).

p M. Baba, M. Saitoh, K. Taguma, K. Shinohara, K. Yoshido, Y. Semba, S. Kasahara, N. Nakayama, H. Goto, T. Ishimoto and U. Nagashima, J. Chem. Phys. 130, 134315 (2009).

q J. Schiedt and R. Weinkauf, Chem. Phys. Lett. 266, 201 (1997).

r M. Cockett and K. Kimura, J. Chem. Phys. 100, 3429-3441 (1994).

s J. Ferguson, L. Reeves and W. Schneider, Can. J. Chem. 35, 1117-1123 (1957).

t J. Warren, J. Hayes and G. Small, Chem. Phys. 102, 323-336 (1986).

" EELS; P. Swiderek, M. Michaud, G. Hohlneicher and L. Sanche, Chem. Phys. Lett. 176, 289-284 (1991).

v J. Piest, J. Oomens, J. Bakker, G. von Helden and G. Meijer, Spectrochim. Acta A 57, 717-735 (2001). 
Figure 1

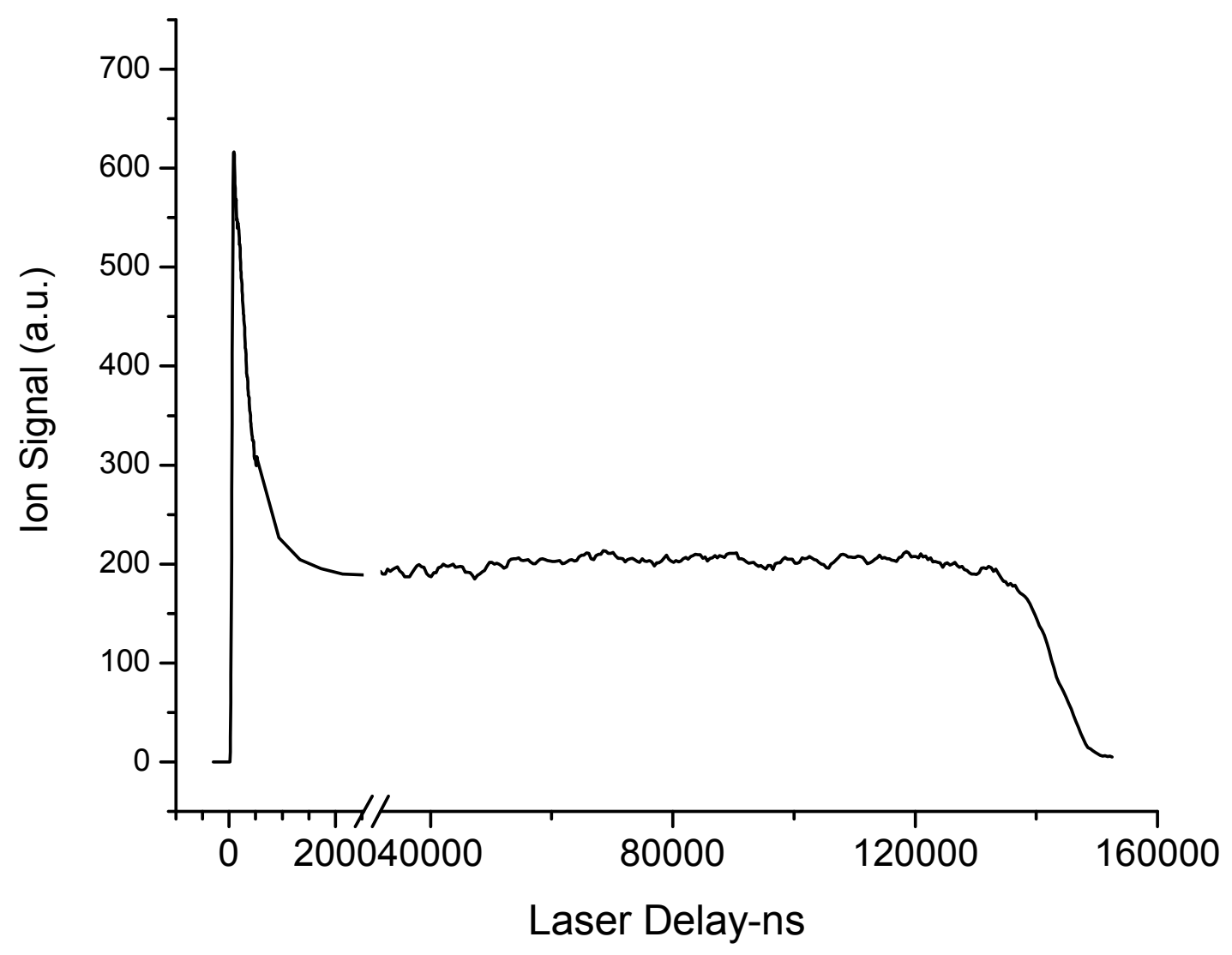

Figure 1 A time-delay scan of the pump-probe ionization of naphthalene excited to the $8^{1}$ vibration of $S_{1}$ and ionized at a later time by $193 \mathrm{~nm}$ photons. An initial population is composed of both singlets and triplets. The singlet component decays with the fluorescence lifetime, while the triplet signal persists until the excited sample passes the detection region. There is a time scale change after the singlet decay to accommodate the much longer triplet lifetime. 
Figure 2

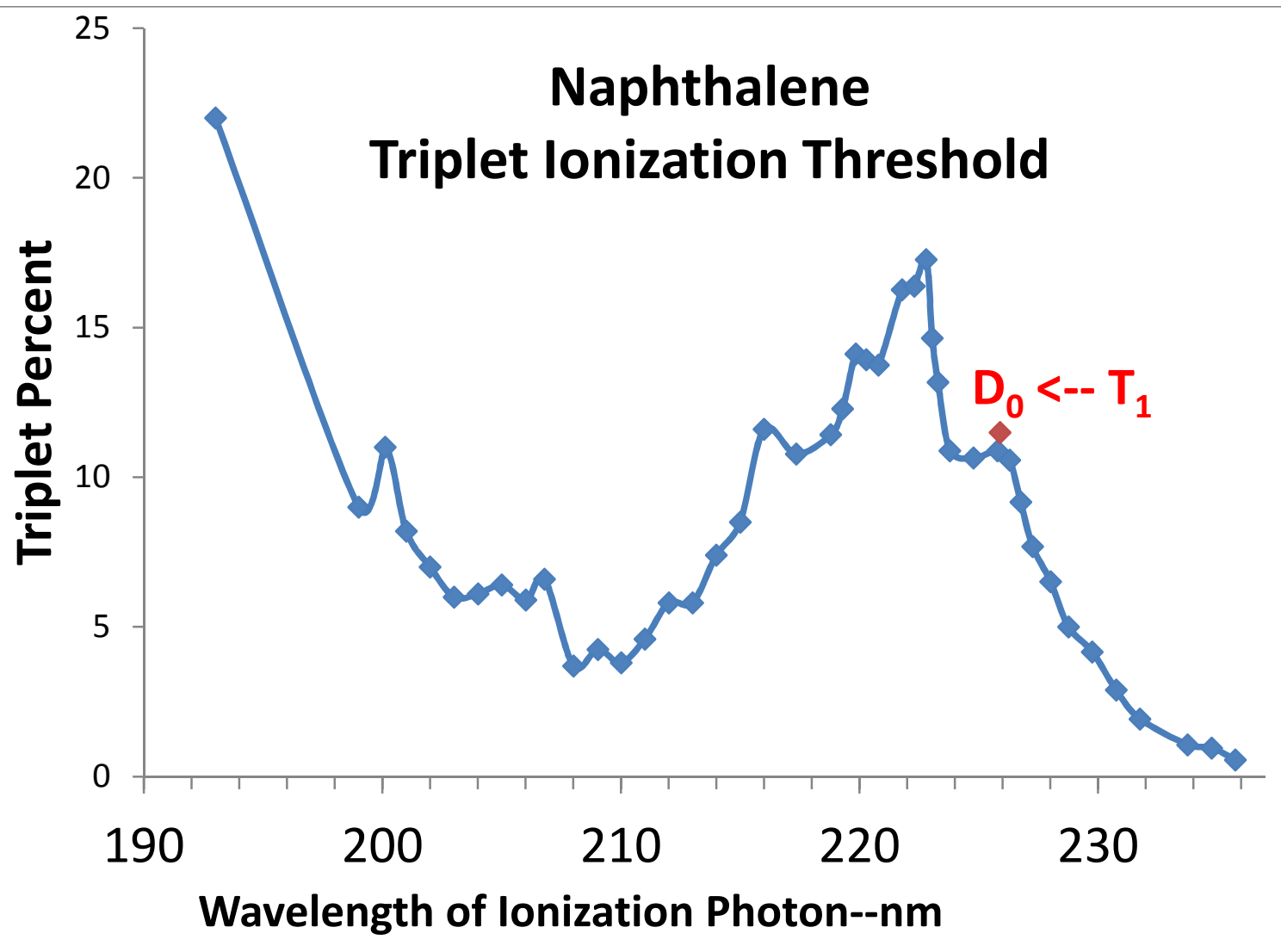

Figure 2: Ratio of the naphthalene triplet ionization signal to the total ionization signal when the pump and probe lasers are overlapped in time, as a function of the wavelength of the probe laser. The vibrationless $\mathrm{T}_{1}$ ionization energy is shown. 
Figure 3

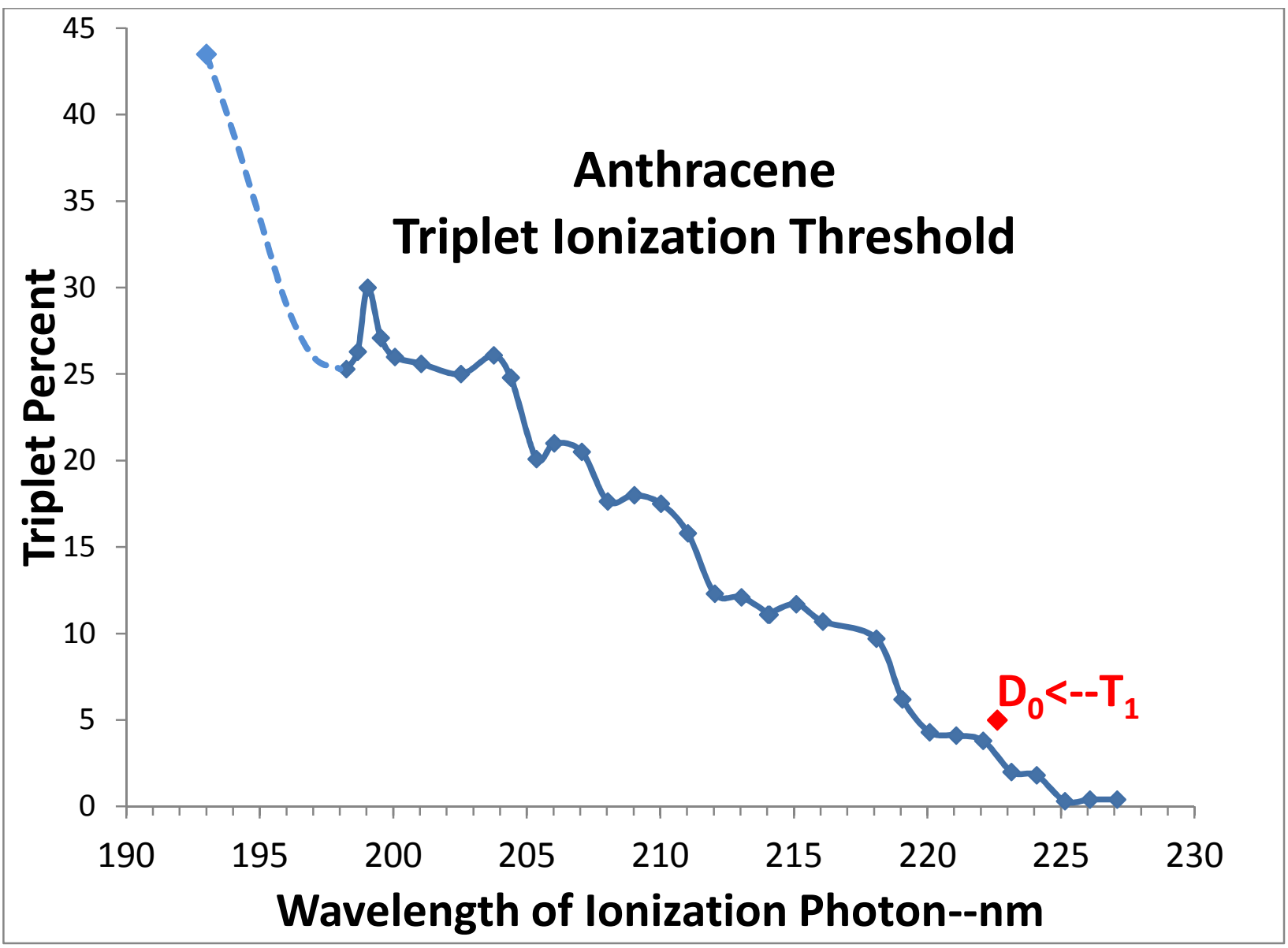

Figure 3 As in figure 2, but for anthracene triplet. 
Figure 4

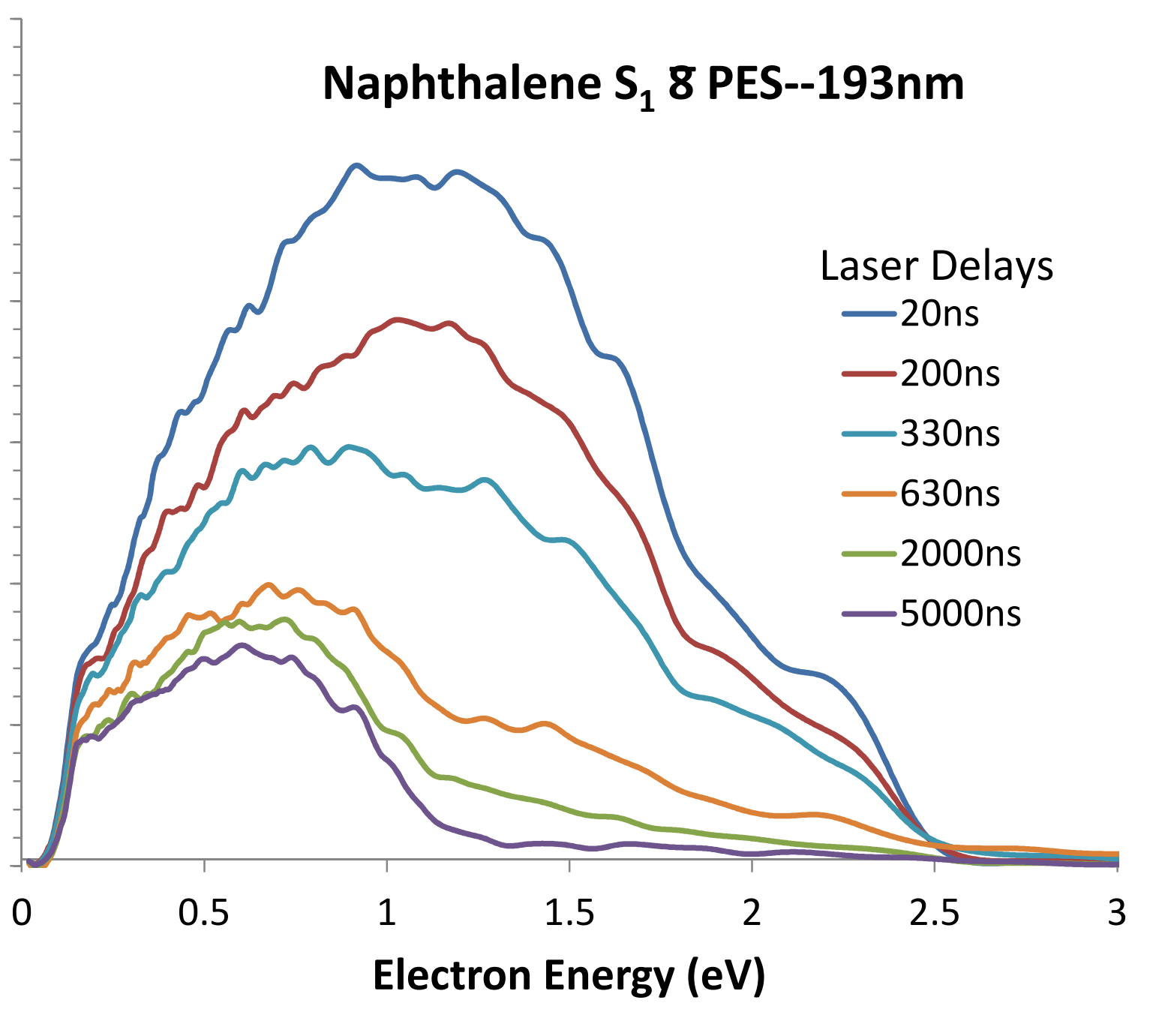

Figure 4 The pump-probe photoelectron spectrum of naphthalene excited to the $8^{1}$ vibration of $S_{1}$, and ionized with $193 \mathrm{~nm}$ photons at various different pump-probe decays. The singlet undergoes transitions to both the ground and first excited states of the ion, and the signal decays exponentially to the residual triplet signal seen at long delay time. 
Figure 5
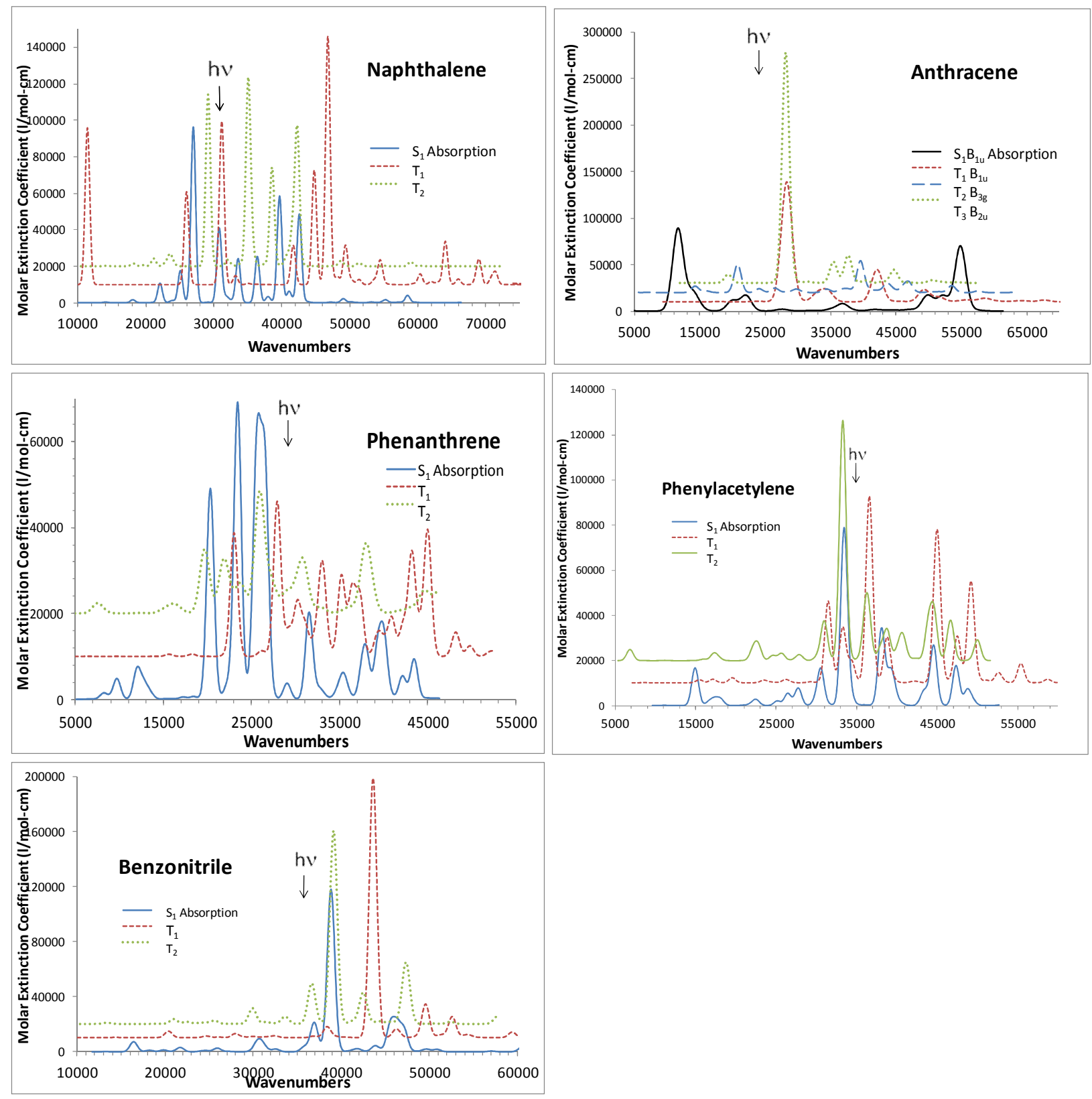

Figure 5 Absorption spectra for the lowest singlet state and lower triplet states of naphthalene, anthracene, phenanthrene, phenylacetylene, and benzonitrile calculated using the symmetry adapted cluster-configuration interaction method. The energies of photons resonant with $\mathrm{S}_{1}-\mathrm{S}_{0}$ are indicated. To reduce congestion, spectra are offset from one another by 10000 molar extinction coefficient units. Absorption values are calculated from state oscillator strengths using Gaussian line shapes of $800 \mathrm{~cm}^{-1}$ width. 
Figure 6

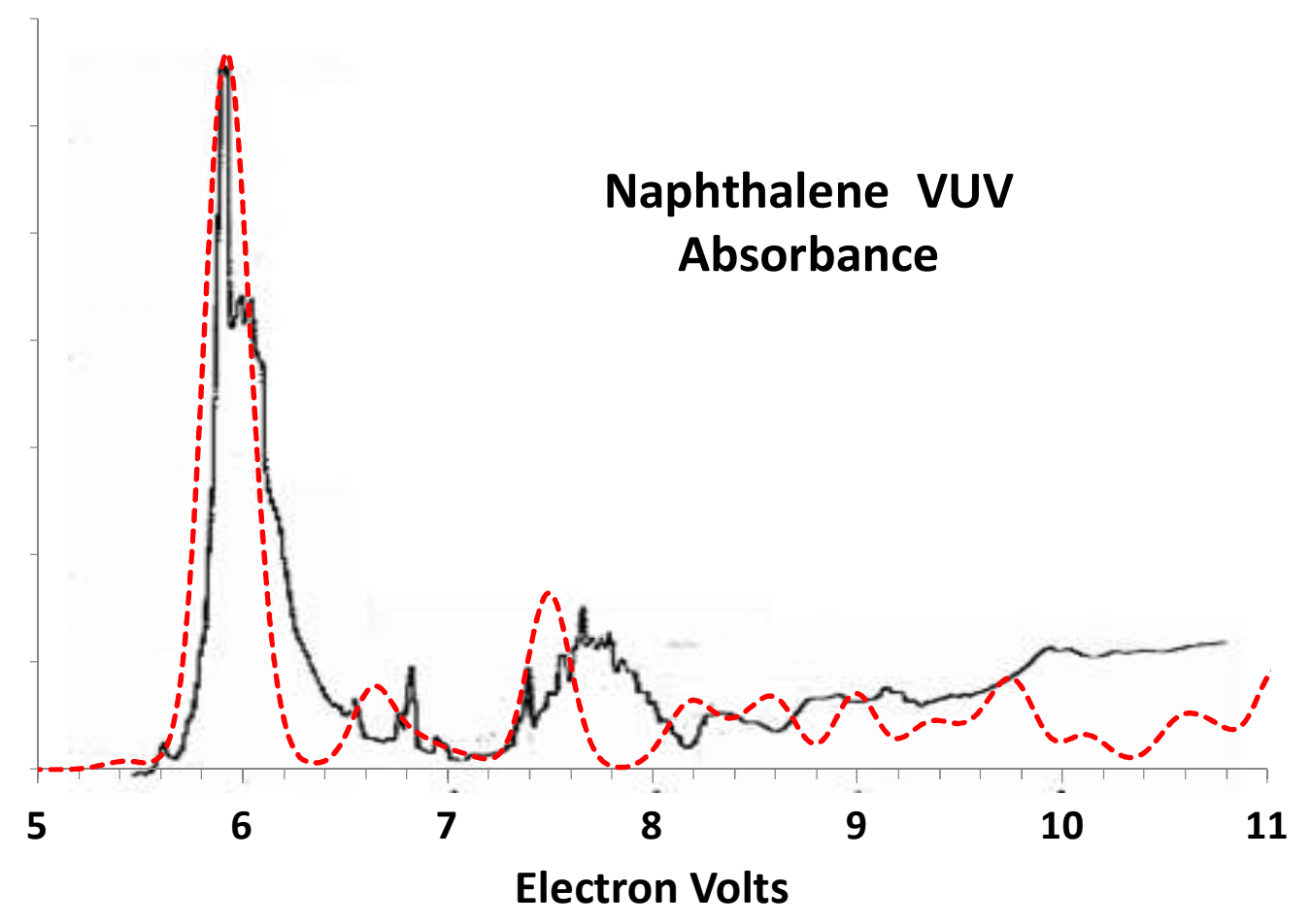

Figure 6: A comparison between the experimental vacuum ultraviolet absorption spectrum of Koch, Otto, and Radler (solid line), and a spectrum derived from SAC-Cl calculations (dashed line). 
Figure 7

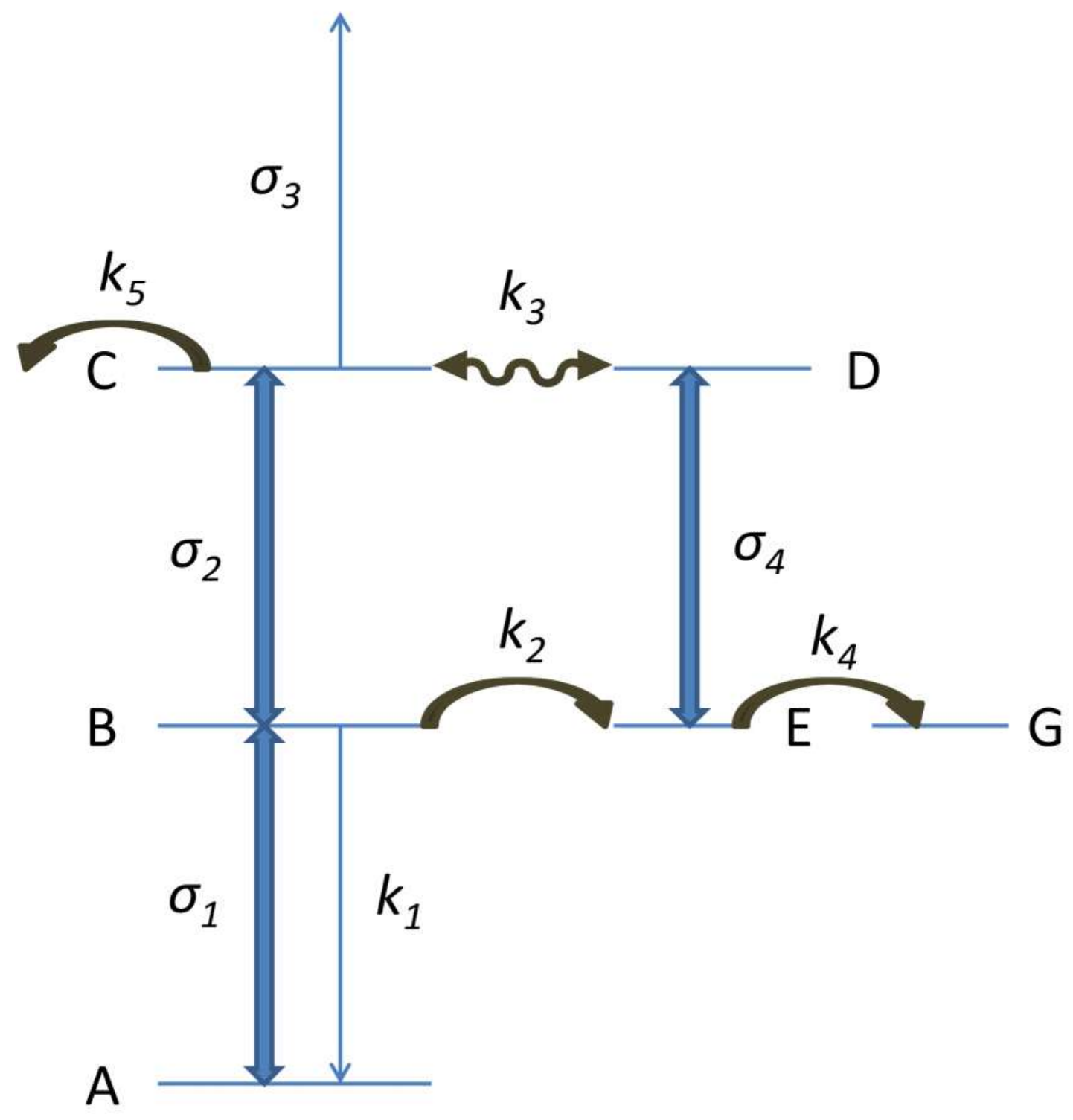

Figure 7: The multistate kinetic scheme used to model the population dynamics of isolated polycyclic aromatic molecules irradiated by a nanosecond laser pulse. Sigmas represent absorption and stimulated emission cross-sections, while k's are the rates of various intrinsic relaxation processes. State labels are characterized in the text. 
Figure 8

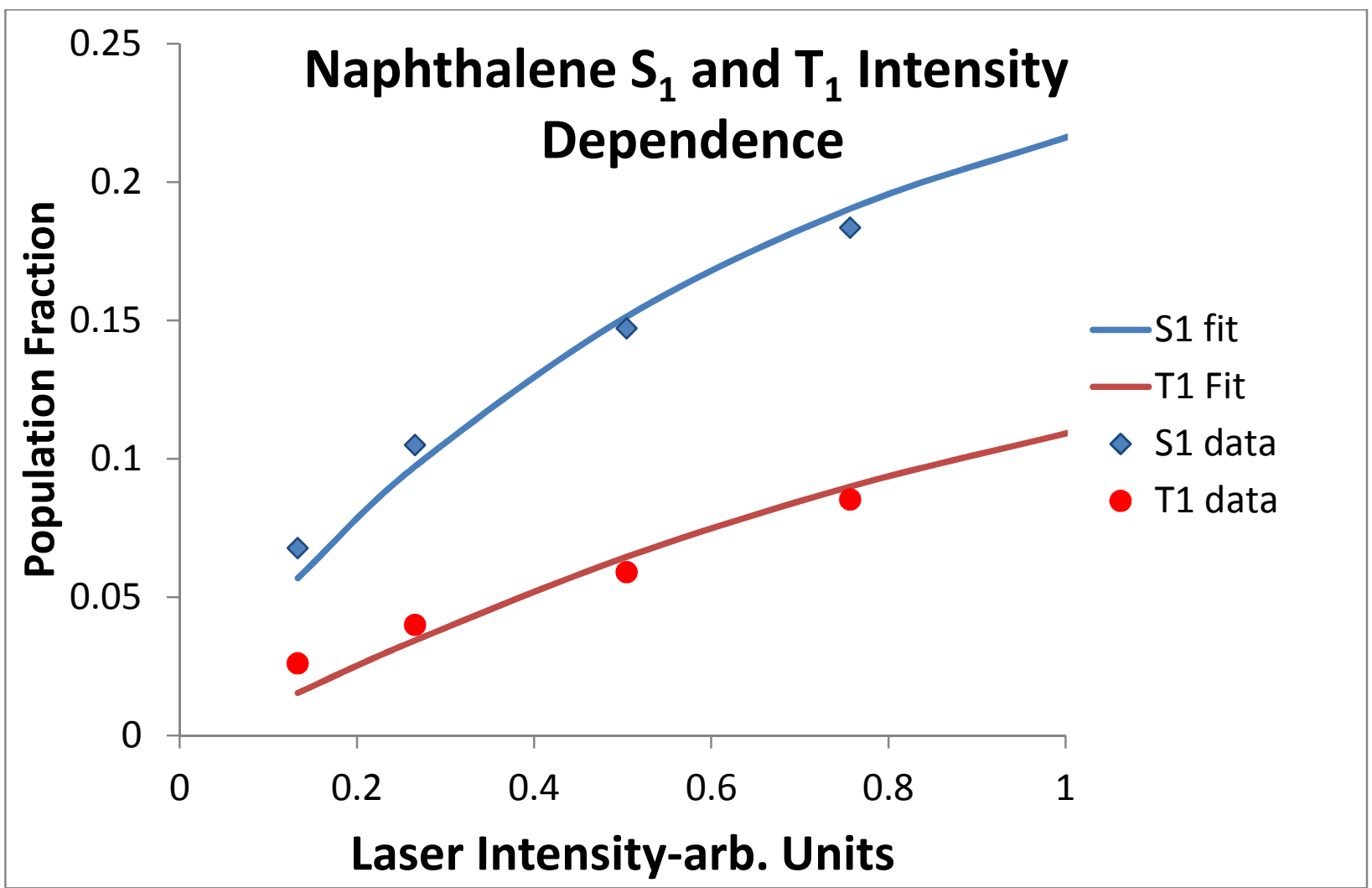

Figure 8: Data points are relative singlet and triplet populations of naphthalene as a function of relative laser intensity. The highest laser intensity is assigned a value of one. Solid lines are calculated using the multistate kinetic scheme of figure 7, with the parameters described in the text. To place the data points on the figure, the singlet population at the highest intensity is made equal to the calculated population fraction. 
Figure 9

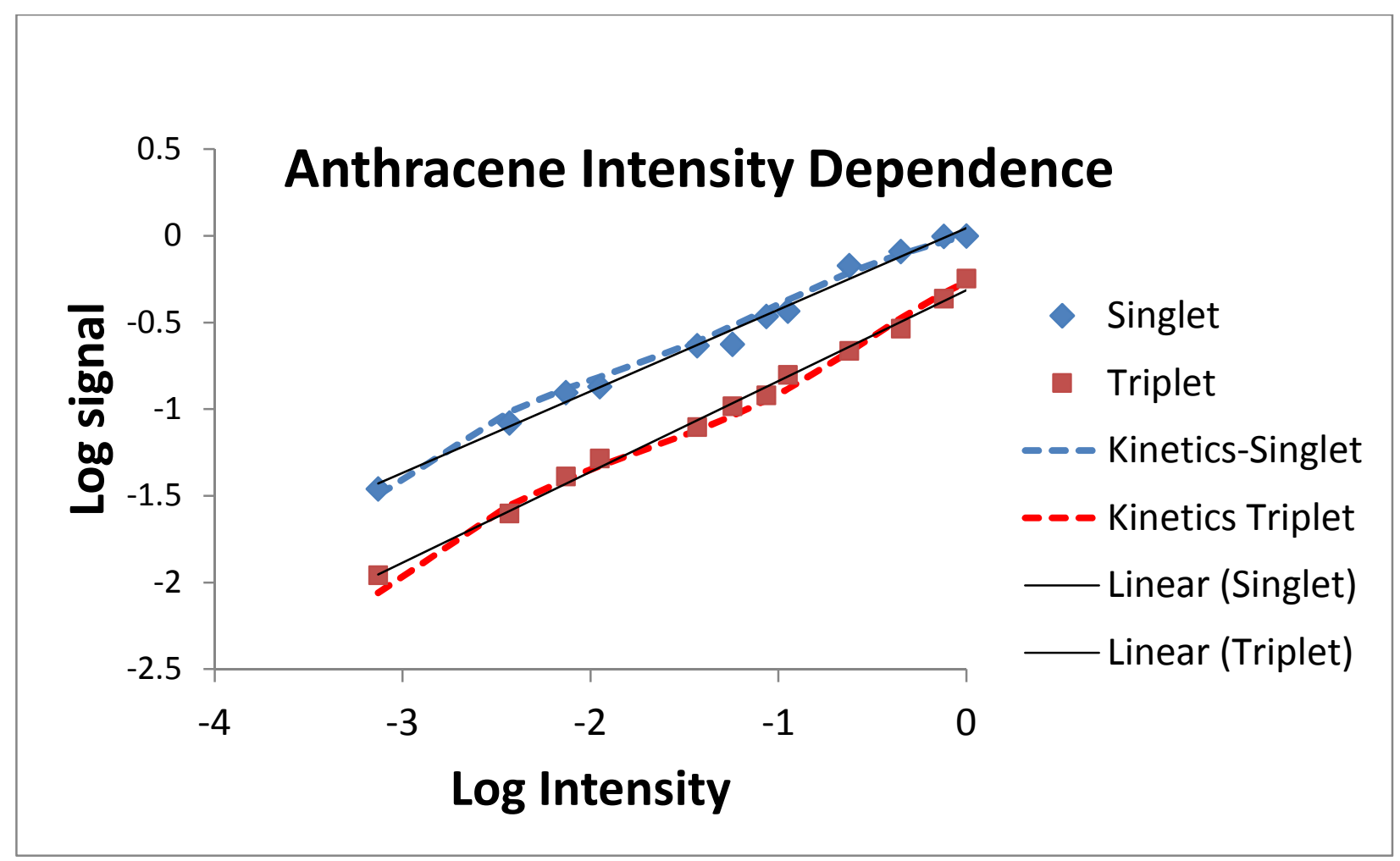

Figure 9: As in Figure 8, but a log-log plot for anthracene. Linear least-squares fits to the logarithmic data points are shown by the solid black lines. 


\section{REFERENCES}

${ }^{1} \mathrm{~A}$ good entry into the extensive early literature on radiationless transitions can be found in the review by J. Kommandeur, W. Majewski, W. L. Meerts, and D. Pratt, Ann. Rev. Phys. Chem. 38, 433-462 (1987).

2 G. W. Robinson, Excited States, ed. E. C. Lim, Academic (New York), 1-34 (1974).

3 J. Jortner, S. A. Rice, and R. Hochstrasser, Adv. Photochem, ed. J. N. Pitts Jr., Interscience (New York) 149-309 (1969).

${ }^{4}$ M. A. Duncan, T. Dietz, M. Liverman, and R. E. Smalley, J. Phys. Chem, 85, 7-9 (1981).

5 J. L. Knee, C. E. Otis, and P. M. Johnson, J. Phys. Chem, 87, 2232-2239 (1983).

${ }^{6}$ T. Dietz, M. A. Duncan, and R. E. Smalley, J. Chem. Phys, 76, 1227-1232 (1982).

7 J. L. Knee and P. M. Johnson, J. Chem. Phys. 80, 13 (1984).

${ }^{8}$ A. Sur and P. M. Johnson, J. Chem Phys. 84, 1206 (1986).

${ }^{9}$ T. Dietz, M. A. Duncan, A. Puiu, and R. E. Smalley, J. Phys. Chem. 86,4026-4029 (1982).

10 J. Knee and P. M. Johnson, J. Phys. Chem. 89, 948 (1985).

${ }^{11}$ A. Doi, S. Kasahara, H. Kato and M. Baba, J. Chem. Phys 120, 6439-6448 (2004).

${ }^{12}$ M. Okubo, M. Mosono, J. Wang, M. Baba, and H. Kato, J. Chem. Phys. 116, 9293-9299 (2002).

${ }^{13}$ K. Yoshida, Y. Semba, S. Kasahara, T. Yamanaka, and M. Baba, J. Phys. Chem. 130, 194304/1-9 (2009).

${ }^{14}$ M. Baba, M. Saitoh, K. Taguma, K. Shinohara, K. Yoshida, Y. Semba, S. Kashahara, N.

Nakayama H. Goto, T. Ishimoto, and U. Nagashima, J. Chem Phys. 130, 134315/1-9 (2009).

15 J. Hofstein, H. Xu, T. Sears, and P. M. Johnson, J. Phys. Chem. A, 112, 1195-1201 (2008).

${ }^{16}$ P. M. Johnson and T. Sears, J. Phys. Chem. A 117, 7786-7793 (2013).

${ }^{17}$ D. Parker, R. Minns, T. Penfold, G. Worth, and H. Fielding, Chem. Phys. Lett. 469, 43-47 (2009).

${ }^{18}$ R. Minns, D. Parker, T. Penfold, G. Worth, and H. Fielding, Phys. Chem. Chem. Phys. 12 1560715615 (2010).

${ }^{19}$ D. Zakheim and P. Johnson, Chem. Phys. 46, 263-272 (1980).

${ }^{20}$ C. M. Marian, WIREs Comput. Mol. Sci. 2, 187-203 (2012). 
${ }^{21}$ B. Henry and W. Siebrand, Chem. Phys. Lett. 3, 90-92 (1969).

22 H. Agren, B. Minaev, and S. Knuts, J. Phys. Chem. 98, 3943-3949 (1994).

${ }^{23}$ T. Penfold and G. Worth, Chem. Phys., 375, 58-66 (2010).

${ }^{24}$ G. George and G. Morris, J. Mol. Spectr., 26, 67-71 (1968).

${ }^{25}$ E. Koch, A. Otto and K. Radler, Chem. Phys. Lett. 16, 131-135 (1972).

${ }^{26}$ E. Koch, A. Otto and K. Radler, Chem. Phys. Lett. 21, 501-505 (1973).

27 D. Dempster, T. Morrow and M. Quinn, J. Photochem. 2, 329-341 (1973).

${ }^{28}$ D. P. Craig and I. G. Ross, J. Chem. Soc. 1954, 1589-1606 (1954).

${ }^{29}$ R. Compton, K. Grattan and T. Morrow, J. Photochem. 14, 61-66 (1980).

30 P. Johnson, M. Berman and D. Zakheim, J. Chem. Phys. 62, 2500-2502 (1975).

${ }^{31}$ P. Johnson, Acc. Chem. Res. 13, 20-26 (1980).

32 P. Johnson and C. Otis, Ann. Rev. Phys. Chem., 32, 139-157 (1981).

${ }^{33}$ D. Lubman and M. Kronick, Anal. Chem., 54, 660-665 (1982).

${ }^{34}$ S. Okajima, B. Forch, and E. C. Lim, J. Phys. Chem. 87, 4571-4573 (1983).

35 B. Shore, "The Theory of Coherent Atomic Excitation", John Wiley \& Sons, New York 1990, p. 1151.

${ }^{36}$ B. Shore, Ibid. Chapter 22.

${ }^{37}$ F. Lahmani, A. Tramer, and C. Tric, J. Chem. Phys. 60, 4431-4447 (1974).

38 P. C. Haarhoff, Mol. Physics, 7, 101-117 (1964).

${ }^{39}$ R. Englman and J. Jortner, Mol. Physics, 18, 145-164 (1970).

${ }^{40}$ B. Henry, Acct. Chem. Res., 10, 207-213 (1977).

${ }^{41}$ A. Doi, S. Kasahara, H. Kato, and M. Baba, J. Chem. Phys. 120, 6439-6448 (2004).

${ }^{42}$ C. Schick and P. Weber, J. Phys. Chem. A, 105, 3725-3734 (2001); N. Kuthirummal and P. Weber, J. Molec. Struct. 787, 163-166 (2006). 